\title{
Video Article \\ Creation of a Rodent Model of Abdominal Aortic Aneurysm by Blocking Adventitial Vasa Vasorum Perfusion
}

\author{
Hiroki Tanaka ${ }^{1,2}$, Naoki Unno ${ }^{2}$, Tatsuro Yata ${ }^{2}$, Hirona Kugo ${ }^{3}$, Nobuhiro Zaima ${ }^{3}$, Takeshi Sasaki ${ }^{4}$, Tetsumei Urano ${ }^{1}$ \\ ${ }^{1}$ Department of Medical Physiology, Hamamatsu University School of Medicine \\ ${ }^{2}$ Division of Vascular Surgery, Second Department of Surgery, Hamamatsu University School of Medicine \\ ${ }^{3}$ Department of Applied Biological Chemistry, Graduate School of Agriculture, Kindai University \\ ${ }^{4}$ Department of Organ \& Tissue Anatomy, Hamamatsu University School of Medicine
}

Correspondence to: Naoki Unno at unno@hama-med.ac.jp

URL: https://www.jove.com/video/55763

DOI: doi:10.3791/55763

Keywords: Medicine, Issue 129, Abdominal aortic aneurysm, vasa vasorum, arteriosclerosis, tissue hypoxia, rodent model, intraluminal thrombus, adipocytes

Date Published: 11/8/2017

Citation: Tanaka, H., Unno, N., Yata, T., Kugo, H., Zaima, N., Sasaki, T., Urano, T. Creation of a Rodent Model of Abdominal Aortic Aneurysm by Blocking Adventitial Vasa Vasorum Perfusion. J. Vis. Exp. (129), e55763, doi:10.3791/55763 (2017).

\section{Abstract}

The adventitial vasa vasorum (VV) provides oxygen and nourishment to the aortic wall. Hypoxia in the aortic wall can cause enlarged abdominal aortic aneurysms (AAAs). This article introduces and describes a standard protocol used to induce AAAs through adventitial VV hypoperfusion created with a combination of polyurethane catheter insertion into the aortic lumen and suture ligation of the infrarenal abdominal aorta.

The protocol involves the use of male rats weighing 300-400 g, which are provided food and water ad libitum. After laparotomy with a ventral midline abdominal incision, exfoliation of the aorta is performed, which blocks blood flow from the perivascular tissue. Aortotomy involving a small incision adjacent to the renal artery branches is performed, and a polyurethane catheter is inserted using an 18-gauge indwelling needle. After repairing the incision, tight ligation of the aorta over the catheter blocks $\mathrm{V} V$ blood flow from the proximal direction through the aortic wall without disturbing the aortic blood flow. This technique can induce an AAA with progressive aortic dilatation.

The greatest benefit of this model is that VV hypoperfusion causes tissue hypoxia and the development of an infrarenal AAA, which has morphological and pathological characteristics similar to those of a human AAA.

\section{Video Link}

The video component of this article can be found at https://www.jove.com/video/55763/

\section{Introduction}

The abdominal aorta is composed of the following three layers: the inner vascular wall (intima), medial layer (media), and outer vascular wall (adventitia), and of these, the adventitia has a unique blood supply system known as the vasa vasorum (VV). Aortic tissue is supplied with oxygen through adventitial VV perfusion and simple oxygen diffusion from the aortic blood flow ${ }^{1}$. However, geographically, the abdominal aorta has the least distribution of VVs compared to that in other parts of the aorta. ${ }^{2}$

A previous study reported on tissue hypoxia in human abdominal aortic aneurysm (AAA) walls with thick intraluminal thrombus (ILT) ${ }^{3}$. Additionally, it has been shown that an adventitial VV in aneurysmal walls is occluded with arteriosclerotic changes at a significantly higher rate, which is associated with tissue hypoxia in the AAA walls ${ }^{4}$. Based on these findings, a novel rodent model of AAA was created by inducing adventitial VV hypoperfusion ${ }^{5}$. In this model, VV hypoperfusion caused tissue hypoxia and the development of an infrarenal AAA, which had morphological and pathological characteristics similar to those of a human AAA ${ }^{6}$. Prime examples were the presence of ILTs and the accumulation of hyperplastic adipocytes ${ }^{6}$, and the potential to cause rupture ${ }^{7,8}$. These findings have been rarely observed in previous rodent models. Therefore, this model may greatly contribute to a deeper understanding of the mechanism responsible for AAA development and rupture. We introduce and describe a standard protocol used to induce AAAs through adventitial VV hypoperfusion, and we explain how to induce hypoxia in the aortic wall using surgical techniques.

\section{Protocol}

Animal care and experiments were performed in accordance with the guidelines of the Hamamatsu University School of Medicine Animal Care Committee at the Center for Animal Care. 


\section{Surgical Procedure for Creating the Model}

NOTE: Place the surgical instruments into a bead sterilizer for $10 \mathrm{~s}$ preoperatively. Use sterile gloves intraoperatively.

1. Use male rats weighing $300-400 \mathrm{~g}$. Allow the rats access to food and water ad libitum.

2. Anesthetize the rat with isoflurane inhalation $(2.0-3.0 \mathrm{~mL} / \mathrm{L})$. Confirm proper anesthetization by toe pinch.

3. Shave hair on the abdomen with an electric razor, and scrub the abdomen with alcohol and a povidone-iodine solution.

4. Place the rat in the supine position on the operation table. Use vet ointment on the rat's eyes to prevent dryness while under anesthesia.

5. Perform a laparotomy with a ventral midline abdominal incision using a scissors. To secure a clear surgical field, pack the abdominal contents inside the abdominal cavity with sterile gauze using a wound retractor.

6. To detach the aorta from the perivascular tissue, gently pick up and tear the retroperitoneum with tweezers to expose the aortic wall and exfoliate the infrarenal aorta in the retroperitoneal space from the level of the left renal vein to the bifurcation from the perivascular tissue (Figure 1A).

7. Ligate the vessels branching from the abdominal aorta with a 5-0 silk string to block blood supply at a point away from the aorta that does not narrow the aortic lumen.

8. To block aortic blood flow, place vascular clips just below the renal artery and just above the bifurcation of the aorta NOTE: The size of the clips should be larger than the aortic diameter to block the blood flow completely. A temporary 5-0 silk string ligature is also sufficient for blocking blood flow instead of the clips.

9. Similar to creating a dot hemorrhage on the surface of the aortic wall, cut the anterior aortic wall using a micro-scissor $5 \mathrm{~mm}$ distally from the aortic clip, adjacent to the renal artery branches, to insert a polyurethane catheter.

10. Insert a polyurethane catheter (outside diameter $0.55 \mathrm{~mm}$, inside diameter $0.37 \mathrm{~mm}$ ) using a 24-gauge indwelling needle shallowly through the incision to wash away blood in the aorta with water. Administer a 1-mL syringe filled with water into the polyurethane catheter, and wash away the remaining blood in the aorta with water. After irrigation, remove the catheter from the aorta.

11. Before inserting a polyurethane catheter (outside diameter $1.20 \mathrm{~mm}$, inside diameter $0.94 \mathrm{~mm}$ ) using an 18-gauge indwelling needle, cut the polyurethane catheter to $10 \mathrm{~mm}$ long (Figure 1B).

1. Insert the cut piece of the polyurethane catheter into the aortic lumen from the incision made in step 1.9. Completely place the 10-mm catheter into the aortic lumen, and reposition the midpoint of the catheter to match the location of the incision (i.e., cover the incision with the catheter).

12. Repair the incision with interrupted sutures using an 8-0 monofilament string (Figure 1C).

13. Ligate the abdominal aorta using a combination of 5-0 silk string and the polyurethane catheter (Figure 1D).

NOTE: The aortic lumen can be maintained by the indwelling catheter. Ligate the aorta firmly to prevent misaligning the catheter position. The surgeon's knot is recommended, because it adds an extra twist when tying the first throw of the knot, forming a double overhand knot, and an additional turn provides more friction and can reduce loosening.

14. After ligation, remove the vascular clip at the aortic bifurcation, and then, remove the vascular clip below the renal artery to restore the antegrade blood flow. Confirm gross aortic pulsation.

15. Close the abdominal incision in two layers, the peritoneum and other layers, with a 4-0 polypropylene suture. Tightly suture the incision to prevent a protruded organ.

16. Apply topical lidocaine on the abdominal incision. Place the rat on a heating pad until consciousness returns. Do not leave a rat unattended until it has regained sufficient consciousness to maintain sternal recumbency. Do not return a rat that has undergone operation to the company of other rats until it has fully recovered.

17. Monitor the rat closely after the operation. Inject a bolus of sterile saline $(1.0-2.0 \mathrm{~mL})$ subcutaneously if a rat shows signs of distress or body weight loss.

18. Postoperatively, observe the time-course development of the enlarged aorta on an ultrasound echogram. Measure the maximum diameter from the inside edge to the outside edge of the abdominal aorta as described ${ }^{5}$.

\section{Harvesting, Fixing, and Elastica-van Gieson (EVG) Staining}

1. Twenty-eight days postoperatively, administer sodium pentobarbital $\geq 100 \mathrm{mg} / \mathrm{kg}$ intraperitoneally to euthanize the rats.

2. Cut open the rat's abdominal cavities ventrally. Make the initial incision using a scalpel. Harvest the aneurysm using scissors and place the harvested aortas in $10 \%$ neutrally buffered formalin for $24-48 \mathrm{~h}$.

NOTE: The surgeon must be careful to prevent injury to the aorta and other organs when exposing the aneurysm. An elevated abdominal organ should be observed, indicating AAA formation. Figure 2 shows an in vivo image and ex vivo image; this representative sample of AAAs is not ruptured.

3. EVG staining

\section{Solution Preparation}

1. Prepare Verhoeff's hematoxylin by mixing the following in the given order: alcoholic hematoxylin $20 \mathrm{~mL}, 10 \%$ ferric chloride 8.0 $\mathrm{mL}$, and Lugol's iodine $8 \mathrm{~mL}$. Mix the solution between each addition. This solution can be prepared fresh as needed.

2. Prepare Van Gieson's solution by mixing $1 \%$ acid fuchsin $1 \mathrm{~mL}$ and saturated picric acid $45 \mathrm{~mL}$. Allow to stand overnight and mix well. This solution is stable for 2 weeks.

3. Prepare differentiating solution ( $2 \%$ ferric chloride) by mixing $10 \% \mathrm{FeCl}_{3} 10 \mathrm{~mL}$ in $40 \mathrm{~mL}$ distilled water. This solution may be prepared fresh as needed.

4. Prepare $5 \%$ hypo by dissolving $5.0 \mathrm{~g}$ sodium thiosulfate in $100 \mathrm{~mL}$ distilled water. This solution is stable for 1 year.

\section{Staining procedure}

NOTE: See reference ${ }^{9}$ for details.

1. Briefly, hydrate the fixed paraffin sections of the harvested aortas in distilled water for 10 min. Place the aortas in Verhoeff's hematoxylin for $25 \mathrm{~min}$. Wash with water and dip 10-30 times in differentiating solution $\left(2 \% \mathrm{FeCl}_{3}\right)$. Rinse in water. Place in $5 \%$ hypo for $1 \mathrm{~min}$. Wash in water. Place in Van Gieson's solution for $5 \mathrm{~min}$ 
2. Dehydrate in alcohol in the following order: $75 \%, 90 \%, 95 \%, 100 \%$, and $100 \%$ alcohol for 30 s each. Then, clear in xylene for 5 min, twice. Place on a coverslip with resinous mounting medium.

NOTE: Elastic fibers appear blue-black to black; nuclei: blue to black; collagen: red; and other tissue elements: yellow.

\section{Representative Results}

The described operative techniques create a novel animal model of a chronic aortic hypoxia-induced aneurysm by using a combination of polyurethane catheter insertion and suture ligation of the infrarenal abdominal aorta in rats. The rats described in the Protocol section were euthanized 28 days after the procedure. The aortas were harvested and imaged to visualize aneurysm formation. Figure 2 shows development of the fusiform AAA. The upper and lower ends of the aorta in ex vivo have a normal diameter without dilatation. Aortic diameters were measured using transabdominal ultrasonography (Figure 3). The diameter generally reaches its maximum size at about 14 days after the procedure; thereafter, it remains unchanged or slightly increases. Figure $\mathbf{4}$ shows the histopathological image of the aneurysm at its maximum diameter after EVG staining. The image of the tissue on day 28 (aneurysm) showed prominent degradation of elastic fibers compared to that on day 0.
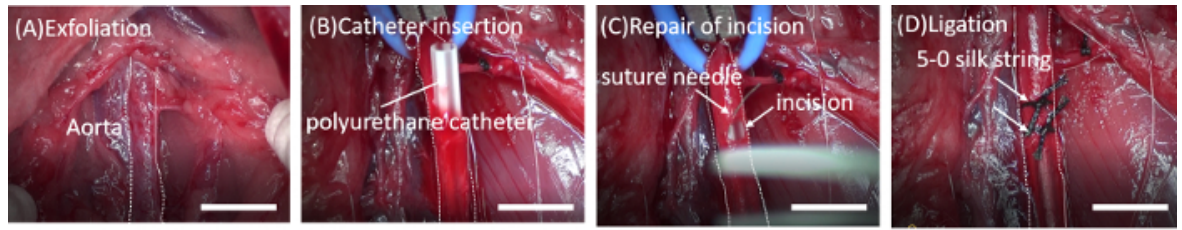

Figure 1: Surgical procedures to induce an abdominal aortic aneurysm (AAA).

(A) The infrarenal aorta is exfoliated from the surrounding tissue. (B) A polyurethane catheter cut $10 \mathrm{~mm}$ long is inserted through a small incision in the aorta. (C) The incision is repaired with an 8-0 monofilament suture and blood flow is restored. (D) The aorta is ligated with a 5-0 silk suture over the inserted catheter. Scale bar $=5 \mathrm{~mm}$. Please click here to view a larger version of this figure.
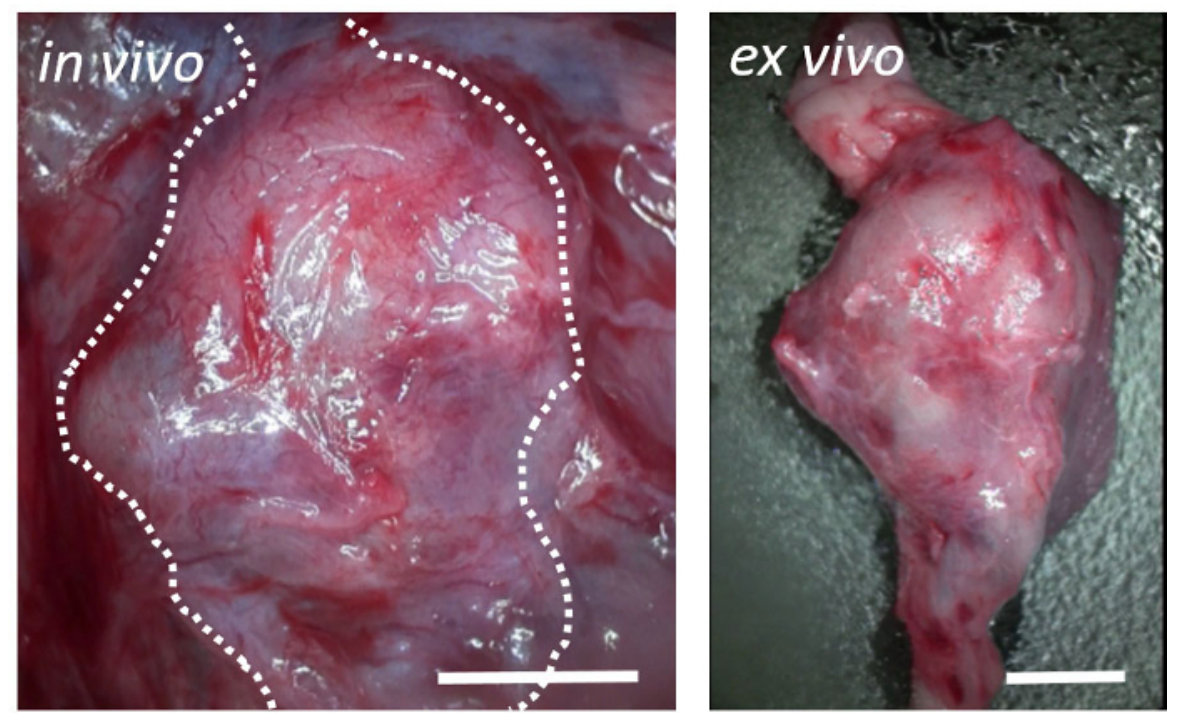

Figure 2: Postoperative representative results.

Macroscopic view on postoperative day 28 showing development of fusiform abdominal aortic aneurysms. The elevated margin of the retroperitoneum corresponds to the outer edge of the aneurysm (broken lines; left). The upper and lower ends of the aorta in ex vivo are normal (right). Scale bar $=3 \mathrm{~mm}$. Please click here to view a larger version of this figure. 


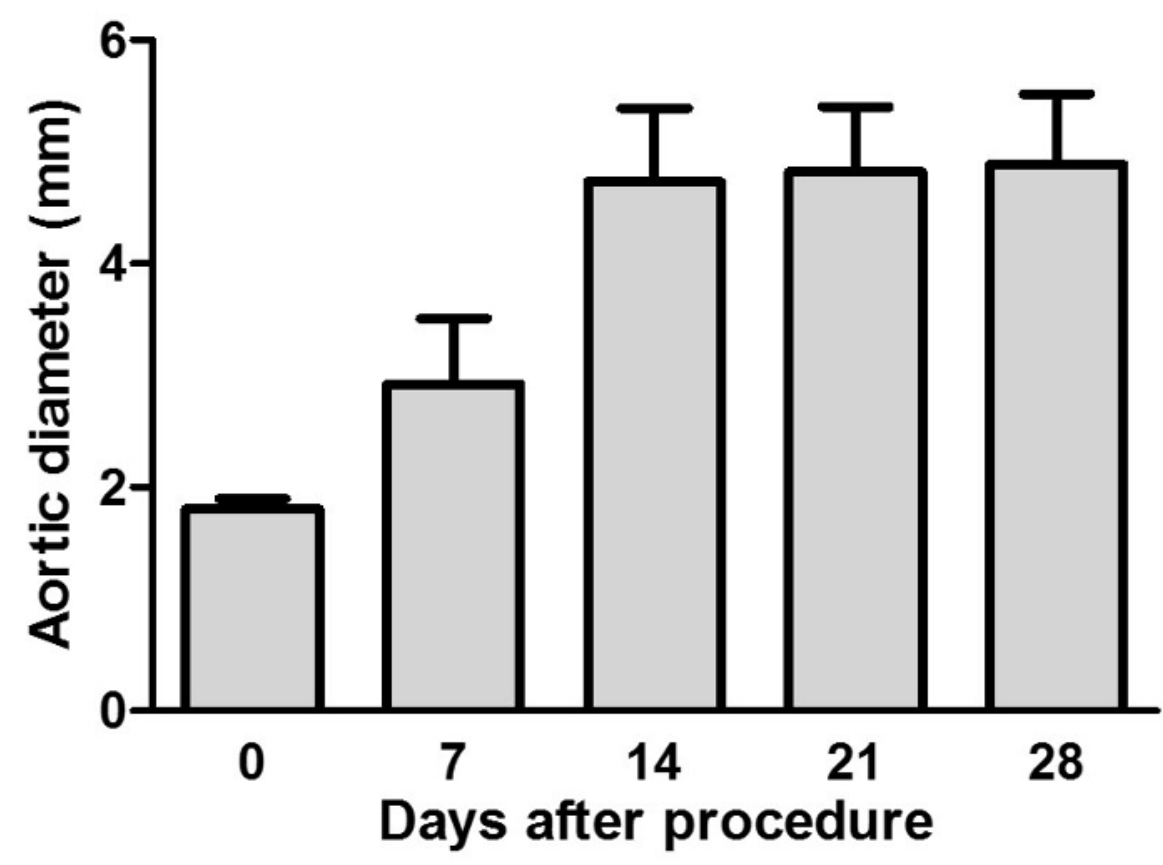

Figure 3: Maximum aortic diameters measured using transabdominal ultrasonography.

The aortic diameter steadily increased in this rat model. Aortic diameters are presented as a mean \pm standard deviation $(n=12)$. Please click here to view a larger version of this figure.

Day 0

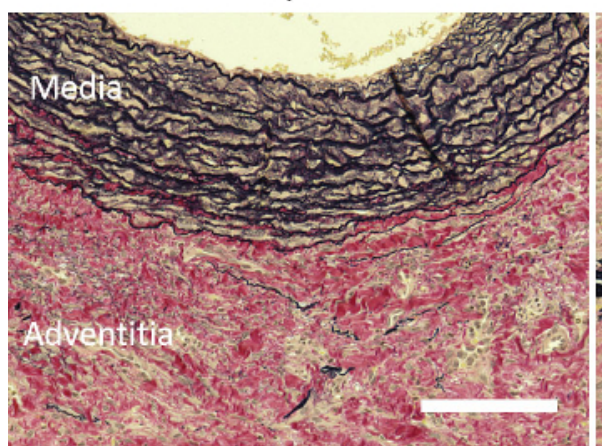

Day 28 (Aneurysm)

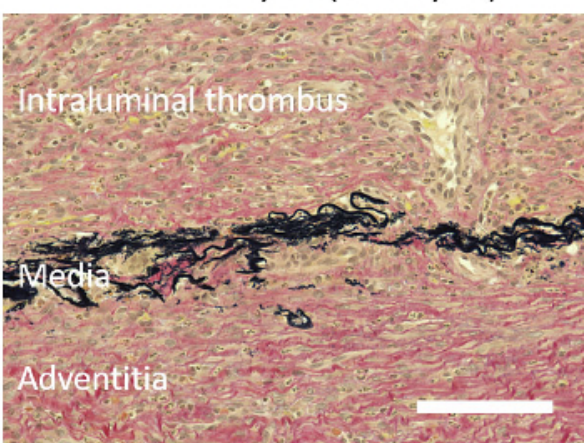

Figure 4: Representative images of aneurysmal tissue with Elastica van Gieson staining.

Histological evaluation with EVG staining showing the degenerative elastic lamina in the media and formation of an intraluminal thrombus 28 days after the procedure (right). Elastic fiber fragmentation in the aortic media and sparse collagen fiber in the aortic adventitia are observed on day 28. Day 0 is before the procedure (left). Scale bar $=500 \mu \mathrm{m}$. Please click here to view a larger version of this figure.

\section{Discussion}

Under physiological conditions, the inner layers of the aortic wall are nourished by diffusion from the luminal blood flow, whereas the outer and middle layers are nourished by the $\mathrm{V} V$, which penetrate from the adventitia into the medial $\mathrm{V}^{1}$. VV blood flow into the abdominal aortic wall can originate from the following three directions/areas: (1) the proximal direction through the aortic wall, (2) distal direction through the aortic wall, and (3) perivascular tissues ${ }^{10}$. Previously, our histological analysis of human tissues identified significant stenosis or occlusion of the VV in the AAA wall, suggesting that $\mathrm{VV}$ blood flow into the abdominal aortic wall can be reduced ${ }^{4}$. It is an extremely important point in this protocol that an infrarenal AAA was caused by a combination of polyurethane catheter insertion and suture ligation of the infrarenal abdominal aorta. To carefully exfoliate the tissue layer, surgeons must smoothly insert a polyurethane catheter into the aorta and firmly ligate the aorta to cause chronic hypoxia due to hypoperfusion of the adventitial VV and aneurysm formation. Using these techniques, blood flow in the aortic wall is consequently decreased, and a local hypoxic environment is induced. The blood flow reduction and hypoxia-induced aneurysm formation indicates that $\mathrm{V} V$ blood flow into the abdominal aortic wall plays a role in the pathogenesis of AAA formation.

Specifically, an aortic aneurysm model must satisfy the following conditions: a 1.5-fold increase in the vascular diameter compared to baseline, degeneration of the tunica media, and inflammation of the aortic wall. The most popular animal models have been constructed by inducing inflammatory responses using substances, such as $\mathrm{CaCl}_{2}{ }^{11}$, elastase ${ }^{12}$, and angiotensin $\mathrm{II}^{13}$. These models can have a high reproducibility and obviously cause pathological change, and they have been commonly used in research studies. In our model, we assessed the aortic diameter using ultrasonography every 7 days from before the procedure was performed until day 28 after the procedure (Figure 3). Results showed that the aortic diameter moderately increased over the 28 days, indicating that this change in the diameter is similar to that in previous rodent 
models. Gross observation of the vascular form indicated a smooth fusiform shape (Figure 2). On day 28, we sacrificed the rats and performed histopathological analysis of the aortic tissue that was recovered. Tearing and disappearance of the elastic and collagen fibers of the tunica media and adventitia were observed (Figure 4). Moreover, inflammatory cells, such as macrophages, were present from the tunica adventitia to the tunica media.

Currently, the treatment options available for AAAs are limited to surgical repair and endovascular stent grafting, with mortality rates of 30 - $50 \%$ in patients with AAA rupture ${ }^{14}$. However, no drug has been approved for clinical use to treat AAAs. There is debate that there are discrepancies in the pathological findings between humans and established animal models used in AAA research. Similarities in the pathogenesis between human AAA and animal AAA models are essential for the development of pharmacological treatments. Regarding the effectiveness of rodent models, our rat model is morphologically similar to humans in terms of intraluminal thrombus ${ }^{5}$ and adipogenesis ${ }^{8}$. Furthermore, about $20 \%$ of the rats in this study had AAA rupture and died within 28 days after the procedure. Although aortic aneurysm rupture is the most critical event for this disease, rupture is uncommon with established experimental AAA models, and the mechanism has not yet been elucidated. Therefore, this model is useful for understanding the mechanism of dilation of the aortic diameter and rupture of the aneurysm.

The creation of this model is required for some surgical procedures. Therefore, researchers must practice creating this model, which is a limitation of this model. In the future, we would like to create a rodent model in which we can decrease blood flow by gradually thickening the $\mathrm{V} V$ walls, resulting in spontaneous aortic aneurysm.

\section{Disclosures}

The authors have nothing to disclose.

\section{Acknowledgements}

This work was supported by Grants-in-Aid for Scientific Research (B) (20291958) to N.U.; Grants-in-Aid for Young Scientists (A) (25713024) to N.Z.

\section{References}

1. Wolinsky, H., Glagov, S. Comparison of abdominal and thoracic aortic medial structure in mammals. Deviation of man from the usual pattern. Circ Res. 25 (6), 677-686 (1969).

2. Sano, M., et al. Lymphangiogenesis and angiogenesis in abdominal aortic aneurysm. PLoS One. 9 (3), e89830 (2014).

3. Vorp, D. A., et al. Association of intraluminal thrombus in abdominal aortic aneurysm with local hypoxia and wall weakening. $J$ Vasc Surg. $\mathbf{3 4}$ (2), 291-299 (2001).

4. Tanaka, H., et al. Adventitial vasa vasorum arteriosclerosis in abdominal aortic aneurysm. PLoS One. 8 (2), e57398 (2013).

5. Tanaka, H., et al. Hypoperfusion of the Adventitial Vasa Vasorum Develops an Abdominal Aortic Aneurysm. PLoS One. 10 (8), e0134386 (2015).

6. Tanaka, H., et al. Imaging Mass Spectrometry Reveals a Unique Distribution of Triglycerides in the Abdominal Aortic Aneurysmal Wall. $J$ Vasc Res. 52 (2), 127-135 (2015).

7. Kugo, H., et al. The preventive effect of fish oil on abdominal aortic aneurysm development. Biosci Biotechnol Biochem. 80 (6), 1186-1191 (2016).

8. Kugo, H., et al. Adipocyte in vascular wall can induce the rupture of abdominal aortic aneurysm. Sci Rep. 631268 (2016).

9. Lichtenberg, A., Cebotari, S., Tudorache, I., Hilfiker, A., Haverich, A. Biological scaffolds for heart valve tissue engineering. Methods Mol Med. 140 309-317 (2007)

10. Heistad, D. D., Marcus, M. L., Larsen, G. E., Armstrong, M. L. Role of vasa vasorum in nourishment of the aortic wall. Am J Physiol. $\mathbf{2 4 0}$ (5), H781-787 (1981).

11. Yoshimura, K., et al. Regression of abdominal aortic aneurysm by inhibition of c-Jun N-terminal kinase. Nat Med. 11 (12), $1330-1338$ (2005)

12. Azuma, J., Asagami, T., Dalman, R., Tsao, P. S. Creation of murine experimental abdominal aortic aneurysms with elastase. J Vis Exp. (29) (2009).

13. Lu, H., et al. Subcutaneous Angiotensin II Infusion using Osmotic Pumps Induces Aortic Aneurysms in Mice. J Vis Exp. (103) (2015).

14. United Kingdom, E. T. I., et al. Endovascular versus open repair of abdominal aortic aneurysm. N Engl J Med. 362 (20), 1863-1871 (2010). 